Nonetheless, there are reports that mouse homolog of Blimp-1 is expressed in the myotome ${ }^{15}$, and it will be interesting to determine whether it functions as a slow muscle selector there as well.

1. Baxendale, S. et al. Nat. Genet. 36, 88-93 (2004)

2. Devoto, S.H. et al. Development 122, 3371-3380 (1996).

3. Weinberg, E.S. et al. Development 122, 271-280
(1996)

4. Lewis, K.E. et al. Dev. Biol. 216, 469-480 (1999).

5. Barresi, M.J. Stickney, H.L. \& Devoto, S.H. Development 127, 2189-2199 (2000).

6. Currie, P.D. \& Ingham, P.W. Nature 382, 452-455 (1996).

7. Blagden, C.S. et al. Genes Dev. 11, 2163-2175 (1997).

8. Wolff, C., Roy, S. \& Ingham, P.W. Curr. Biol. 13, 1169-1181 (2003).

9. Turner, C.A. Jr., Mack, D.H. \& Davis, M.M. Cell 77, 297-306 (1994).
10. Shapiro-Shelef, M. et al. Immunity 19, 607-620 (2003).

11. Roy, S., Wolff, C. \& Ingham, P.W. Genes Dev. 15 1563-1576 (2001)

12. Schebesta, M., Heavey, B. \& Busslinger, M. Curr. Opin. Immunol. 14, 216-223 (2002).

13. Brent, A.E. \& Tabin, C.J. Curr. Opin. Genet. Dev. 12 548-557 (2002).

14. Sacks, L.D. et al. Development 130, 3391-3402 (2003).

15. Chang, D.H., Cattoretti, G. \& Calame, K.L. Mech. Dev. 117, 305-309 (2002)

\title{
Two Pax are better than one
}

\section{Richard S Mann}

In fly eye development, new experiments suggest that two distinct $P a x$ genes control tissue growth and identity, respectively. Notably, these two functions may be encoded by distinct isoforms of the human gene PAX6

Most biologists, and many nonbiologists, are probably familiar with the experiment in which scientists generated fruit flies that had more than the normal pair of compound eyes $^{1}$. This result, produced by the forced misexpression of the gene eyeless (ey; called PAX6 in humans), was so notable that it found its way to the front page of The New York Times with the headline "Science Outdoes Hollywood", an accolade that has set a new standard for genetics research ${ }^{2}$. Since this first set of discoveries, the mechanism underlying the control of eye development by the master control gene ey has been the subject of intense research. How can a single gene instruct the development of such a complex structure as the fly's compound eye? On page 31 of this issue, a report by Maria Dominguez and her colleagues ${ }^{3}$ provides new insight into this problem.

\section{Sizing up the eyes}

To generate a complex organ, such as a compound eye, the correct cell types must be formed and these cell types must be correctly organized. In other words, an organ-specific identity must be generated. ey (or Pax6), together with a cohort of subordinate transcription factors, is essential for this aspect of eye development ${ }^{4}$. Organ size must also be controlled, and size control has to be coordinated with the process of organ-identity specification. For the fly eye, there is some

Richard S. Mann is in the Department of Biochemistry and Molecular Biophysics, Center for Neurobiology and Behavior, Columbia University, 701 West $168^{\text {th }}$ Street, HHSC 1104, New York, New York 10032, USA.

e-mail: rsm10@columbia.edu controversy as to whether growth and identity are controlled by the same pathway or by different pathways. Most researchers agree that growth of the eye is controlled by the Notch signal transduction pathway, which is activated during eye development at the interface between the dorsal and ventral halves of the developing eye, also called the dorsal-ventral organizer $^{5-7}$. Some researchers have suggested that Notch also controls eye identity and $e y$ expression $^{8,9}$. Others, however, argue that Notch does not control ey expression or eye identity, only the growth of the eye ${ }^{10}$. That Notch is not responsible for eye identity makes more sense, because this pathway is used not only in the eye but repeatedly during development in a wide variety of contexts. But these results raise an important question: if not $e y$, what are the Notch targets that mediate its growth-promoting activity in the eye?

The new work by Dominguez et al. ${ }^{3}$ answers this question. Their experiments suggest that growth and identity specification are separable pathways in fly eye development. They identify the Pax6-like gene eyegone $(\text { eyg })^{11}$ as the Notch target responsible for mediating its growth-promoting effects in the eye (Fig. 1). Consistent with this notion, in the absence of eyg, the eye primordium does not grow, even though ey is still expressed. This is notable, in part because eyg is not expressed throughout the eye but seems to be

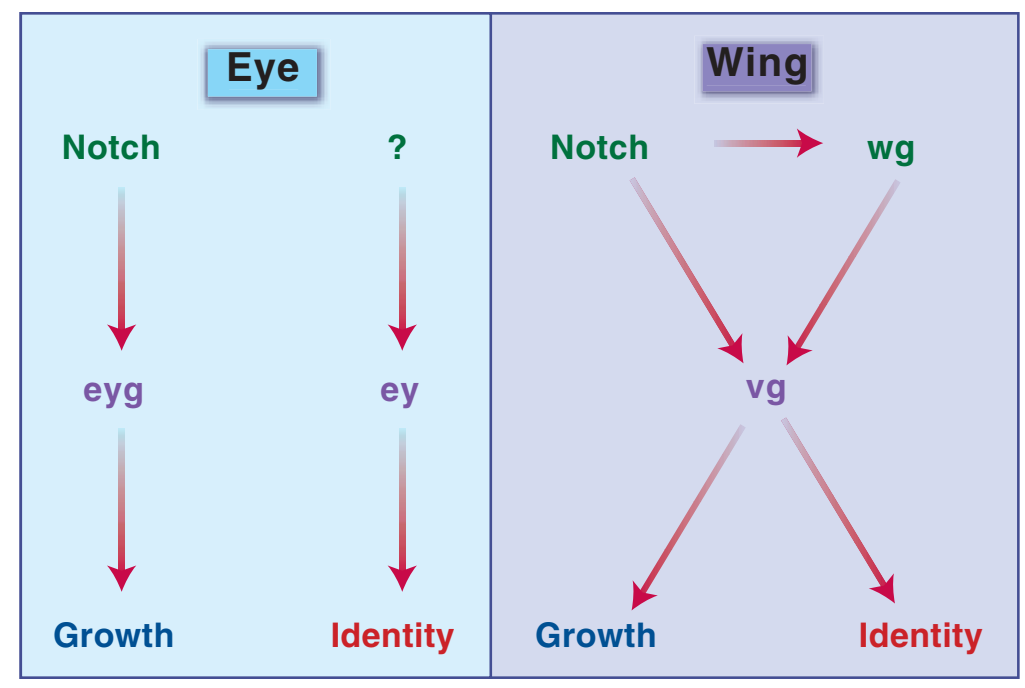

Figure 1 In the fly eye, tissue growth is regulated by Notch and its target eyg. Eye identity is regulated by ey. In the wing, the gene vg carries out both functions and is regulated by both the Notch and Wingless $(\mathrm{Wg})$ pathways. 
specifically induced at the dorsal-ventral organizer. Also, the analysis of mosaic eyes indicates that the requirement for eyg function is not evenly distributed throughout the eye: $e y g^{-}$clones grow poorly when they arise close to the organizer but grow better when they arise far from the organizer. In addition, Dominguez et al. ${ }^{3}$ show that a block in eye growth caused by compromising the Notch pathway can be rescued by forcing eyg expression. Notably, ey was relatively ineffective at rescuing growth in this assay. Thus, eyg seems to be specifically induced by Notch at the dorsal-ventral organizer but required for the growth of the entire eye.

\section{A pair of paired domains}

The story gets even more interesting. Both $e y$ and eyg encode transcription factors of the paired family, which means they have two DNA-binding domains: a homeodomain and a paired domain ${ }^{11}$. Most paired domains, like the one in $e y$, have two subdomains that each have DNA-binding potential. The eyg paired domain, however, has only one of these two subdomains. There does not seem to be an eyg ortholog in humans. But the single PAX6 gene in humans produces two isoforms by alternative splicing ${ }^{12}$. One isoform, PAX6, is similar to Ey, with a homeodomain and a complete paired domain. The other isoform, PAX6(5a), is similar to Eyg, with an intact homeodomain but only one of the two paired subdomains. Dominguez et al. ${ }^{3}$ show that PAX6(5a) induces extensive proliferation without eye differentiation when expressed in the fly wing primordium. The PAX6 isoform induces eye differentiation but not growth. Thus, Dominguez et al. ${ }^{3}$ suggest a model with a division of labor between these two paired family members. Eyg and PAX6(5a), which have incomplete paired domains and, therefore, probably distinct DNA-binding preferences, mediate tissue growth, whereas Ey and PAX6, which have complete paired domains, are responsible for eye tissue identity. These results contrast with how wing development is thought to occur in the fly ${ }^{6}$. As in the eye, growth of the wing primordium depends on Notch signaling activated at a dorsal-ventral organizer. In the case of the wing, however, the Notch target is the gene vestigial $(v g)$, which probably mediates both tissue growth and wing identity (Fig. 1). Thus, these functions seem to be mediated by a single gene in the wing but by two genes in the eye.

\section{Filling in the blanks}

Although the results presented by Dominguez et al. ${ }^{3}$ support the proposed division of labor by eyg and $e y$, several questions persist. Complicating the situation is the fact that both eyg and ey have very similar and closely linked sister genes, twin of eyegone (toe) and twin of eyeless (toy), respectively ${ }^{4,11}$. These gene duplications, together with a lack of mutations that remove their functions, make it difficult to be certain that the sister genes do not also contribute to growth or eye identity. For example, as shown by Dominguez et al. ${ }^{3}$, some eygclones grow quite large, raising the possibility that toe can partially take its place in some circumstances. In addition, paired family proteins can form homo- and heterodimers $^{13}$, so it is possible that Eyg could function together with Ey and/or Toy to mediate eye growth. One argument against this is the experiment showing that PAX6(5a) can induce overgrowth of the wing. As this happens without eye differentiation, it is unlikely that Ey or Toy is present. But eyg is normally expressed in and required for the development of another part of the fly, a portion of the notum ${ }^{14}$. Thus, there must be other factors, perhaps Ey, Toy or one of their downstream targets, that help distinguish the functions of Eyg in the notum from its growth-promoting functions in the eye.

Despite these complications, Dominguez et al. ${ }^{3}$ make a good case for eyg and ey carrying out two distinct functions in eye development.
Their results suggest that eyg is an organizerinduced transcription factor important for the growth of the entire eye. How exactly eyg promotes growth, especially in cells away from the organizer, is still not clear. One possibility is that eyg is responsible for activating another, still to be identified secreted signal. Another possibility is that the requirement for eyg is transient or that small amounts of Eyg are sufficient to carry out its functions. Ey, on the other hand, together with more downstream transcription factors ${ }^{4}$, would be responsible for specifying tissue identity. The Ey and Eyg pathways probably converge at some point, however, perhaps at some of the same downstream targets. Consistent with this notion, blocking some of the factors downstream of Ey both prevents differentiation and induces proliferation ${ }^{15}$, suggesting that there is communication between the identity- and growth-specifying pathways. Tests of these ideas must wait for the identification of Eyg and Ey targets and an analysis of the genetic network downstream of these two Pax genes.

1. Halder, G., Callaerts, P. \& Gehring, W.J. Science 267, 1788-1792 (1995)

2. Angier, N. With New Fly, Science Outdoes Hollywood. in The New York Times A1 (New York, 1995).

3. Dominguez, M., Ferres-Marco, D., Gutierrez-Aviño, F.J., Speicher, S.A. \& Beneyto, M. Nat. Genet. 36, 31-39 (2004).

4. Desplan, C. Cel/ 91, 861-864 (2001).

5. Papayannopoulos, V., Tomlinson, A., Panin, V.M. Rauskolb, C. \& Irvine, K.D. Science 281, 2031-2034 (1998).

6. Irvine, K.D. \& Rauskolb, C. Annu. Rev. Cell Dev. Biol. 17, 189-214 (2001).

7. Dominguez, M. \& de Celis, J.F. Nature 396, 276-278 (1998).

8. Kumar, J.P. \& Moses, K. Cell 104, 687-697 (2001).

9. Kurata, S., Go, M.J., Artavanis-Tsakonas, S. \& Gehring, W.J. Proc. Natl. Acad. Sci. USA 97, 2117-2122 (2000)

10. Kenyon, K.L., Ranade, S.S., Curtiss, J., Mlodzik, M. \& Pignoni, F. Dev. Cell 5, 403-414 (2003).

11. Jang, C.C. et al. Development 130, 2939-2951 (2003).

12. Epstein, J.A. et al. Genes Dev. 8, 2022-2034 (1994).

13. Wilson, D., Sheng, G., Lecuit, T., Dostatni, N. \& Desplan, C. Genes Dev. 7, 2120-2134 (1993).

14. Aldaz, S., Morata, G. \& Azpiazu, N. Development 130 , 4473-4482 (2003)

15. Pignoni, F. et al. Cel/ 91, 881-891 (1997). 